\title{
Study on the Fatigue Modeling of FRP Composite Materials
}

\author{
Zhou Cuixia ${ }^{1, a}$, Zhou Shiming ${ }^{2, b}$, Peng Yiliang ${ }^{1, c}$, Sun Qing ${ }^{2, d^{*}}$ \\ ${ }^{1}$ Henan Electric Power Survey \& Design Institute, Zhengzhou 450007, China \\ ${ }^{2}$ Department of Civil Engineering, Xi'an Jiaotong University, Xi'an, 710049, China \\ azhoucuixia-heny@powerchina.cn, bzsm.xpyxbd@stu.xjtu.edu.cn, \\ c pyl_ok@126.com, ${ }^{d^{*}}$ sunq@mail.xjtu.edu.cn
}

\begin{abstract}
Keywords: Fatigue life model; Nonlinear regression model; FRP; Genetic Algorithm
Abstract. In this paper, three fatigue models are employed and proposed for modeling the fatigue life of different fiber-reinforced composite material systems. In order to identify the unknown parameters in these models, Genetic Algorithm (GA) is used for estimating its values. This technique is a stochastic process that leads straight to different S-N curves that predicts the trend of the experimental data without the need for any assumptions. The calculation results show that these three models, especially the nonlinear regression model, whose parameters are assigned by using GA are all satisfied with the experimental data, and the average value of RMSE is below 0.1. The method of fatigue damage simulation presented should have a very good application prospect.
\end{abstract}

\section{Introduction}

Composite materials show high specific stiffness and strength, so it is widely used in automotive, aerospace and civil engineering applications up to now. Some of these applications involve components that are yielded by cyclic loading. As a result, fatigue becomes one of the most important research objects that need to be given close attention to by the designer. In general, the $\mathrm{S}-\mathrm{N}$ diagram is frequently used in modeling fatigue life of composite materials, which is extremely explicit and straightforward way to represent experimental fatigue data. A number of different types of S-N curves have been presented in the literature, the most famous being the semi-logarithmic and the logarithmic relations. A new linear damage summation model was first used to evaluate the fatigue behavior of composite materials by Nicholas et $\mathrm{al}^{[1]}$. In another work by Mahadevan ${ }^{[2]}$, a damage accumulation model is presented to describe the degradation of composite materials. Recently methods of intelligent algorithms have been employed for interpretation of fatigue data of composite materials. They can offer a means of dealing with many multivariate problems for which an accurate analytical model does not exist or would be very difficult to develop. For example, aritificial neural networks (ANN) have proved to be very good tools to simulate the fatigue life of composite materials ${ }^{[3-5]}$. Genetic programming has been successfully used as a tool for modeling the fatigue behavior, as presented by Anastasios et $\mathrm{al}^{[6]}$. This paper aims to develop a versatile method to explain the fatigue behavior of FRP composite materials. And its effectiveness is evaluated between different models, such as linear regression and accumulation models that are commonly used for this type of material analysis.

\section{Fatigue of composite material}

Fatigue is the main failure mechanism for structures under cyclic loading. Many experimental studies have been carried out for obtaining the fatigue properties of different types of composite materials. Based on these results, numerous fatigue models have been elicited to describe the relationship between stress and fatigue life. Herein we mainly concern with three models which are linear regression model ${ }^{[7]}$, nonlinear regression model (proposed in this paper) and damage accumulation model ${ }^{[2]}$ respectively. 


\section{Linear regression model.}

Primitively semi-logarithmic equation is applied here to fit the S-N curve. Then a linear relationship between the maximum stress $\mathrm{S}$ and the logarithm of $\mathrm{N}$, the number of load cycles of fatigue failure, is given as

$$
S=a \log (N)+b \text {. }
$$

where $a$ and $b$ are parameters related to material properties.

In the previous equation, the parameters should be determined and then fatigue damage will be predicted after a given number of cycles with damage accumulation model.

\section{Nonlinear regression model.}

In this paper, we also design a new model which named as nonlinear or polynomial model on the basis of linear regression model mentioned before. A nonlinear relationship between the maximum stress $S$ and the logarithm of $N$, the number of load cycles of fatigue failure, is expressed by

$$
S=a[\log (N)]^{2}+b \log (N)+c .
$$

where there are three unknown parameters in Eq.(2). Because this model is much more complex and flexible than Eq.(1), it should be more accurate in simulating the fatigue property of composite materials, also which is verified by the following simulation examples.

\section{Damage accumulation model.}

Mahadevan $^{[2]}$ proposed a versatile damage accumulation model which can accurately explains the rapid damage growth during both the early and final stages of life. The proposed function is of the form

$$
S=q\left(\frac{n}{N}\right)^{a}+(1-q)\left(\frac{n}{N}\right)^{b} .
$$

where $S$ is the normalized accumulated damage; $q, a$ and $b$ are material dependent parameters; $n$ is the number of applied loading cycles; $N$ is the fatigue life at the corresponding applied load level.

The parameters in Eq.(3) are defined in terms of fatigue life of interest as

$$
\begin{aligned}
& q=\frac{A\left(\frac{N_{0}}{N}\right)^{a}}{1-(1-A)\left(\frac{N_{0}}{N}\right)^{a}} . \\
& a=\left(\frac{N}{N_{0}}\right)^{b} \\
& b=\left(\frac{N}{N_{0}}\right)^{g} .
\end{aligned} .
$$

where $N_{0}$ is the reference fatigue life; the parameters $a, b$ and $g$ are material dependent constant value. Regression analysis can be carried out to design the value of $q, a$ and $b$, which can be calculated with Eqs.(4)-(6) in a general way.

\section{Parameter identification for Fatigue model}

As a powerful computational search and optimization tool, GA makes the analogy that survival of the fittest individual to its environment is akin to an optimal design. On account of the multi-agent parallelized stochastic search capability of the GA, this technique can be employed herein for modeling the fatigue behavior of composite materials. The process is introduced as follows: At first, fatigue life cycles from experiment were collected, they were incorporated into the fatigue model formulation. Then, the simulated stress was figured out. During the phase of the GA, the objective function was defined as the sum of differences between the stress of experimental results and the simulated results obtained by GA. Through appropriate iterative circulation, the best individual (parameters) will be eventually obtained.

In this study, a computationally-efficient implementation of GA is improved with the appropriate design of chromosome structure and fitness function. 


\section{Chromosome structure.}

Chromosome structure is largely dependent on the nature of the problem itself. Take Damage accumulation model for example, there are three parameters to be estimated in Eq.(3), that are $q, a$ and $b$ respectively, hence the chromosome representation can be defined as

$\mathrm{Q}_{i}=\left\{q_{i}, a_{i}, b_{i}\right\}, i=1, \ldots, N$.

where $N$ is maximum number of chromosomes, $i$ is the $i$ th individual in chromosome.

\section{Fitness function}

Each chromosome is individually going through the same evaluating exercise. A fitness function is a measuring mechanism that is applied to measure the status of a chromosome. Design principle of the fitness function is to make the value of it greater than zero and relatively moderate. In view of this, the root-mean-square error (RMSE) between the simulated and experimental data is taken as the fitness function, which is given by

$$
\text { Fit }_{i}=\frac{1}{n} \sqrt{{\stackrel{\mathrm{a}}{\mathrm{a}}\left({ }_{i=1}\left(F_{\text {sim }, i}-F_{\mathrm{exp}, i}\right)^{2}\right.}_{\text {. }}} \text {. }
$$

where $n$ is the number of data points, and each simulation/experiment data point is indexed by subscript sim.i / exp, $i$.In order to facilitate the fitness function expression, the RMSE is called “ error" for short in the following paragraphs. The rest parameter's design is revealed in Table 1.

Table 1. Parameter design of GA

\begin{tabular}{cccccc}
\hline Gene type & Population & Selection & Crossover rate & $\begin{array}{c}\text { Mutation } \\
\text { Rate }\end{array}$ & $\begin{array}{c}\text { Termination } \\
\text { rule }\end{array}$ \\
\hline Binary code & 60 & Roulette & 0.85 & 0.01 & $\begin{array}{c}\text { Terminate when } \\
\text { RMSE } \geq 0.08\end{array}$ \\
\hline
\end{tabular}

\section{Experimental data and curve fitting}

In this paper, four different composite material systems tested under different fatigue loading conditions of constant amplitude have been modeled using the aforementioned methods and results are compared.

No.1 Material [8]: GFRP multidirectional specimens cut at $15^{\circ}$ off-axis from a laminate with the stacking sequence: $\left\{0 /( \pm 45)_{2} / 0\right\}_{\mathrm{T}}$.

No.2 Material [9]: GFRP multidirectional laminate with a stacking sequence of $\{90 / 0 /( \pm 45) / 0\}_{C}$. The fatigue data is from tests at compressive-compressive cycle loading.

No.3 Material [10]: Multidirectional glass-epoxy laminate with a stacking sequence:

$\left\{( \pm 45 / 0)_{4} / \pm 45\right]_{\mathrm{T}}$.

No.4 Material [11]: Glass epoxy (S2/5208) unidirectional laminate comprised of eight layers, $\{0\}_{8}$.

Noting the expression in brackets " \{\} ", for example, " $0 /( \pm 45) 2 / 0$ " represents that the material is with fibers at $0^{\circ}$ direction and two stitched layers with fibers in both $45^{\circ}$ and $-45^{\circ}$ directions. Also the subscript of "C" or "T" means that the fatigue data is from tests at tension-tension or compression-compression cycle loading. In the present study, the idea of modeling fatigue life with GA is applied on the four data sets. Fatigue data is considered as pairs of maximum cyclic stress and the corresponding cycles to failure. In order to unify the maximum stress in an appropriate range from 0 to 1 , the stress data is divided by its corresponding maximum stress value. These divided stress values are then called normalized stress in the following.

The fatigue data are simulated by three preceding models, and its parameters of the proposed models are identified by GA. Firstly the accuracy and efficiency of the GA should be tested .Taking the example of parameter identification of nonlinear model when using No.1 material's experimental data, the optimization process of GA in Fig. 1 indicates an approximate descending function including the minimum and average "error". And only within 35 generations the RMSE reaches the lowest value that is 0.0349 . It illustrates the GA is enough efficient with high precision. 
The identified parameters by GA are determined according to the directives of each of the proposed processes, which are tabulated in Table 2. In order to analyze all available data with all methods, the error comparisons are performed for the linear, nonlinear and accumulate models (see Table 3). Based on the comparison, it is found that the "error" results derived from nonlinear model is the most accurate than the other methods illustrating that the parameters in this model do not have its physical meaning, but it is more practical and effective than the other methods.

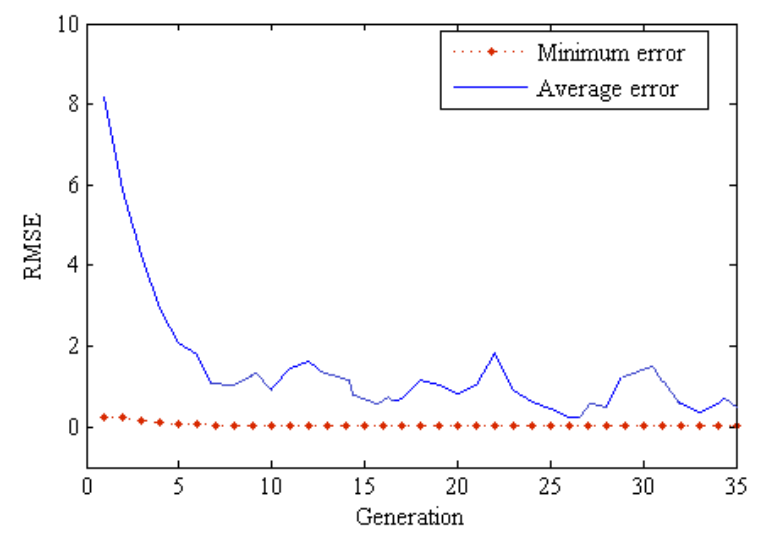

Fig.1. Optimization process of GA

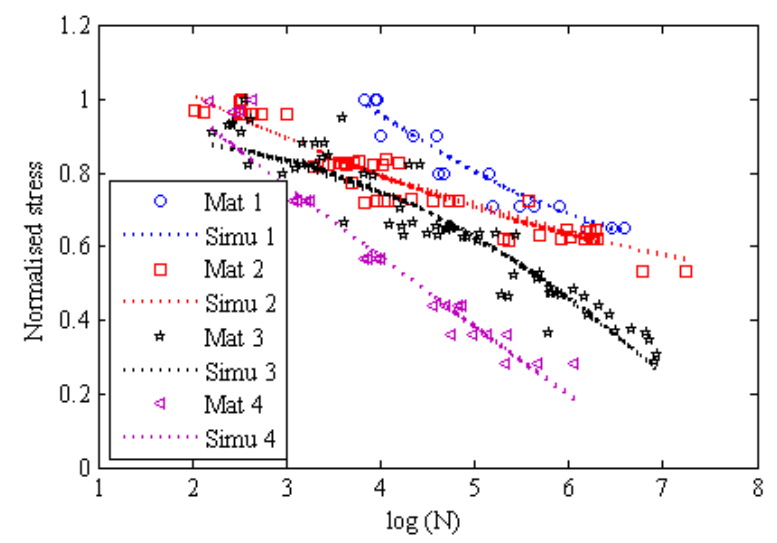

Fig.3. Simulation by nonlinear model

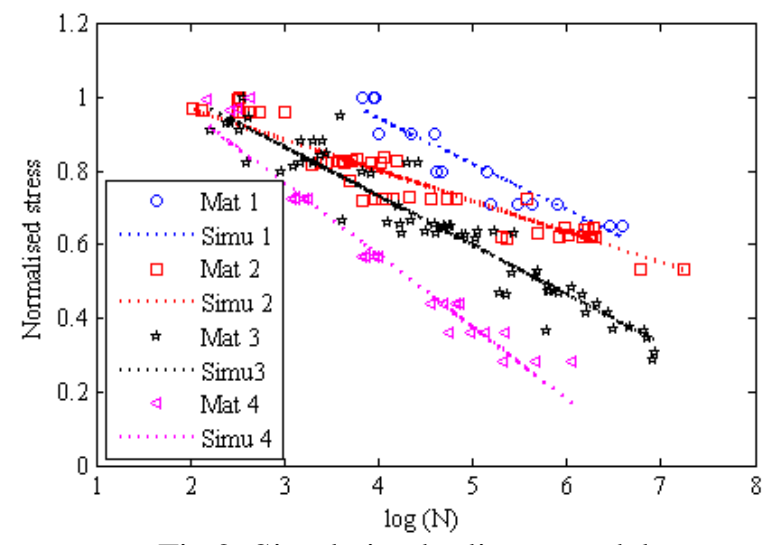

Fig.2. Simulation by linear model

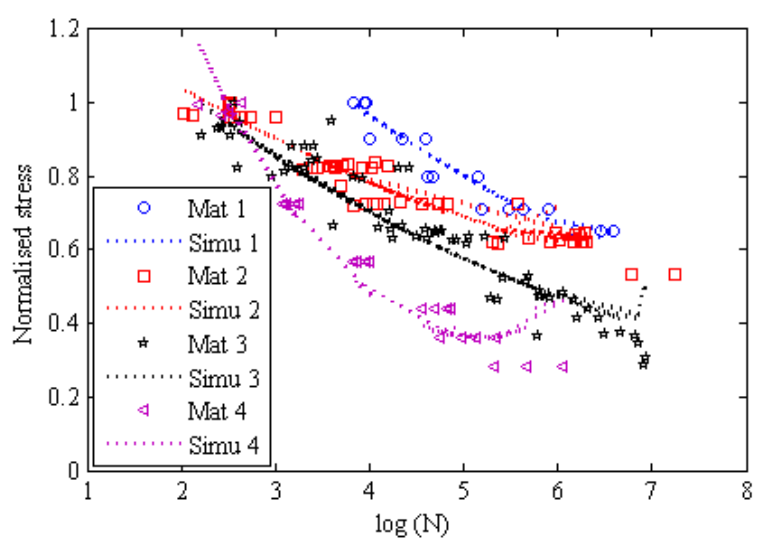

Fig.4. Simulation by accumulate model

Table 2. Calculated fatigue parameters

\begin{tabular}{ccccccccc}
\hline Model $\rightarrow$ & \multicolumn{2}{c}{ Linear model } & \multicolumn{3}{c}{ Nonlinear model } & \multicolumn{3}{c}{ Accumulate model } \\
\cline { 2 - 9 } Materia $_{\downarrow}$ & $\mathrm{a}$ & $\mathrm{b}$ & $\mathrm{a}$ & $\mathrm{b}$ & $\mathrm{c}$ & $\mathrm{q}$ & $\mathrm{a}$ & $\mathrm{b}$ \\
\hline Mat 1 & -0.130 & 1.466 & 0.021 & -0.351 & 2.021 & 0.541 & -0.082 & 1.936 \\
Mat 2 & -0.084 & 1.1387 & 0.009 & -0.164 & 1.315 & 0.503 & -0.064 & 1.063 \\
Mat 3 & -0.123 & 1.219 & 0.003 & -0.162 & 1.330 & 0.389 & -0.085 & 10.000 \\
Mat 4 & -0.203 & 1.383 & 0.002 & -0.210 & 1.373 & 0.180 & -0.160 & -1.624 \\
\hline
\end{tabular}

Table 3 Error comparison with different models

\begin{tabular}{cccccc}
\hline Error $\rightarrow$ & \multicolumn{5}{c}{ RMSE } \\
\cline { 2 - 6 } Model $^{\downarrow}$ & Mat 1 & Mat 2 & Mat 3 & Mat 4 & Average \\
\hline Linear model & 0.0438 & 0.0458 & 0.0684 & 0.0753 & 0.0583 \\
Nonlinear model & 0.0349 & 0.0383 & 0.0680 & 0.0731 & 0.0536 \\
Accumulate model & 0.0419 & 0.0630 & 0.0865 & 0.0485 & 0.0600 \\
\hline
\end{tabular}

Predicted S-N curves from all the available methods are presented in Figs.2-4. It can be concluded that, although based on different approaches, generally speaking all fatigue models have the capability of adequately representing the fatigue properties of the selected experimental data. Herein GA predictions seem to compare favorably with those produced by fatigue models. The simulation 
results prove it superior as it can follow the real trend of the experimental data, without the constraints of a specific equation type.

\section{Conclusions}

GA has been verified to be an extraordinary powerful tool for modeling the behavior of composite specimens subjected to cyclic constant amplitude loading. It can be employed to simulate the fatigue life of different composite material systems. Its unique advantage is that modeling fatigue life by using GA does not need any additional assumptions and the predicted S-N curves do not follow any specific mathematical form. In addition, by comparing the results from linear, nonlinear regression models and damage accumulation model, the nonlinear regression model is the most accurate and effective one which should be used more widely. So it would be a very bright prospect of nonlinear regression model combined with GA for predicting fatigue life of composite material.

\section{References}

[1]. T. Nicholas and S.M. Russ. Elevated-Temperature Fatigue Behavior of Scs-6/Ti-24al-11nb. Materials Science and Engineering a-Structural Materials Properties Microstructure and Processing. (1992), 153(1-2): 514-519.

[2]. H. Mao and S. Mahadevan, Fatigue damage modelling of composite materials. Composite Structures, (2002), 58(4): 405-410.

[3]. R.C.S. Freire, A.D.D. Neto and E.M.F. de Aquino. Building of constant life diagrams of fatigue using artificial neural networks. International Journal of Fatigue, (2005), 27(7): 746-751.

[4]. A.P. Vassilopoulos, E.F. Georgopoulos and V. Dionysopoulos. Modelling fatigue life of multidirectional GFRP laminates under constant amplitude loading with artificial neural networks. Advanced Composites Letters, (2006), 15(2): 43-51.

[5]. A.P. Vassilopoulos, E.F. Georgopoulos and V. Dionysopoulos. Artificial neural networks in spectrum fatigue life prediction of composite materials. International Journal of Fatigue, (2007), 29(1): 20-29.

[6]. A.P. Vassilopoulos, E.F. Georgopoulos and T. Keller. Comparison of genetic programming with conventional methods for fatigue life modeling of FRP composite materials. International Journal of Fatigue, (2008), 30(9): 1634-1645.

[7]. J.N. Yang. Fatigue and Residual Strength Degradation for Graphite-Epoxy Composites under Tension-Compression Cyclic Loadings. Journal of Composite Materials, (1978), 12(Jan): 19-39.

[8]. T.P. Philippidis and A.P. Vassilopoulos. Complex stress state effect on fatigue life of GRP laminates. Part I, experimental. International Journal of Fatigue, (2002), 24(8): 813-823.

[9]. J.F. Mandell and D.D. Samborsky. DOE/MSU composite material fatigue database: test methods, material and analysis. Sandia National Laboratories/Montana State University, SAND97-3002, (2006).

[10]. R.P.L. Nijssen. OptiDAT-fatigue of wind turbine materials database, 2006[J].

[11]. GP, Sendeckyj. Fitting models to composite materials. In: Chamis CC, editors. Test methods and design allowables for fibrous composites, ASTM STP734, American Society for Testing and Materials,(1981), 245-260. 